\title{
L'alimentation méditerranéenne au zénith
}

\author{
Si l'idéal existe, serait-il dans l'alimentation méditerranéenne ? Mais existe-t-elle ? \\ Si elle existe, n'est-elle pas en voie d'extinction, telle une espèce rare ?
}

C ette mise au point se focalise d'abord, avec Éric Birlouez, sur l'analyse de la diversité des modes alimentaires méditerranéens, et ce qui en rend la description difficile. Ces diverses cultures ont néanmoins un point commun qui l'oppose au régime occidental : I'alimentation de terroirs ! Les diètes méditerranéennes ont été "locavores " avant que cela ne devienne une mode. Ils sont aussi caractérisés par une abondance de légumes et de fruits, peu de produits animaux, des produits céréaliers, des légumes secs... ; et selon les lieux une certaine frugalité (régime crétois traditionnel...) des pois chiches et du pain non levé (Égypte, Liban), des escargots (Crête), du poisson (Maroc) : ils partagent en tout cas avec les canons de la diététique la diversité et la variété, bases de l'équilibre alimentaire.

C'est cette diversité qui est sans doute à l'origine des bénéfices santé considérables de l'alimentation méditerranéenne, tant sur le plan cardiovasculaire, que sur le plan cognitif ou des cancers. C'est toute la mise au point d'une des meilleures expertes dans l'épidémiologie de l'alimentation méditerranéenne Mariette Gerber. Ayant longtemps reposé seulement sur des études d'observation convaincantes mais non formelles, l'épidémiologie du régime méditerranéen repose maintenant également sur des études d'intervention telle que Predimed, publiée en 2013. L'intérêt de cette approche épidémiologique est de s'intéresser à un régime global et non pas à un aliment particulier auquel on ne peut attribuer tous les bénéfices d'une alimentation. Longtemps, I'huile d'olive fut emblématique de cette alimentation même si elle n'en était pas le dénominateur commun. De même son intérêt ne réside sans doute pas tant dans ses acides gras, l'acide oléique, que dans ses polyphénols.

Loin de supplanter l'huile d'olive, l'huile d'argan n'en est pas moins un représentant intéressant, bien que de consommation mineure. Ahmed Adlouni, chercheur au Maroc, non seulement y croit mais le démontre avec ses travaux et cette revue de la littérature. L'insaponifiable de cette huile est en effet exceptionnel et rend compte sûrement de la majorité de ses effets dans un grand nombre de fonctions physiologiques. Voici un exemple qui mérite d'être connu.

Pourtant l'alimentation méditerranéenne est menacée de disparition, ceci sera largement évoqué dans d'autres exposés des Entretiens de nutrition de l'Institut Pasteur de Lille sous le pudique vocable de " transition alimentaire ". La France étant un pays du pourtour méditerranéen (le nord fut espagnol jusqu'en 1668 !), il est de notre devoir de la défendre, non pas comme une antiquité mais comme un patrimoine universel et vivant. D'autant que sa disparition s'assortit dans tous les pays du Magreb et ailleurs d'une augmentation considérable de la survenue de diabète, d'obésité, de maladies cardiovasculaires : de quoi se motiver pour se battre sur ce front.

\section{Pr Jean-Michel Lecerf}

Service de Nutrition, Institut Pasteur de Lille 